posed. Tubercle occurs in little rounded masses, whose size rarely exceeds that of a hempseed, and each of these masses has been formed, as it were, from several separate centres; so that we see (in transverse sections through a granulation) what appears to be a section through a small tumour whose elements are more or less concentrically arranged around several centres. This concentric arrangement of elements is frequently absent altogether in cases of fibroid substitution, and is never by any means so marked as in the grey granulation. Where fibroid substitution is commencing in an organ, the new tissue, often starting from the walls of a blood-vessel, proceeds continuously in different directions, and has no tendency to produce rounded masses only, similar to those of tubercle. It often advances rather in the form of bands, and in proportion as this new tissue grows, so do the proper elements of the organ disappear before it; so that at last large tracts of fibro-nuclear and fibre tissue are found in the place of the anatomical elements proper to the part, every vestige of whose original structure has ceased to exist. These are pretty well marked differences as regards (I) the more obvious anatomical peculiarities of the two morbid products, and others even more notable obtrude themselves when we consider (2) their ultimate fate, and (3) their mode of origin. Tubercle always existing in the form of miliary tumours, the more internal elements of each of the component nodules (by the aggregation and union of which a grey granulation is formed), have a strong tendency to undergo fatty degeneration and disintegration-a process which may spread outwards, and ultimately involve the whole nodule*; whilst, with fibroid substitution, it must be clearly understood that the fibro-nuclear condition represents only one temporary stage of this morbid condition; and that, after a time, the number of nuclei gradually diminishes, whilst the fibre-tissue becomes more abundant, and the organ begins to diminish in bulk. No particular tendency to fatty degeneration is evinced; and the rounded and ovoidal nuclei seem for the most part to disappear before the gradually increasing fibre-tissue, just as the proper anatomical elements of the part originally disappeared before the fibronuclear growth. Then, again, tubercle is the anatomical lesion characteristic of a grave constitutional disorder; whilst fibroid substitution seems in most cases to depend far more upon local than upon constitutional causes; and, in those instances where it does seem to be more dependent upon a diathetic condition, this condition is one entirely distinct from tuberculosis.

Several pathological conditions have been described under different names, all of which I am disposed to look upon as fibroid substitutions, whose existence is due more to local than to constitutional causes. Thus, the condition which Laennec described as grey tubercular infiltration, and which is frequently met with surrounding more or less slowly formed caverns in the lung-tissue, was called chronic pneumonia by Chomel, and is still called so by many pathologists, though Addison and others have described it under the simpler name of grey or iron-grey induration. Grisolle and Charcot agree in the opinion that such indurated tissue is due to a new fibre-growth ; the characters of which, moreover, they hold to be precisely similar to that which is met with when what they call chronic pneumonia occurs as an almost immediate sequence of an acute pneumonia. But, seeing that the so-called chronic pneumonia thus occurring, and that met with around old lung-caverns-or even around adventitious products, such as acephalo-cysts-presents similar structural changes, and that these anatomical characters are altogether different from those of acute pneumonia, this name seems an unsuitable one : more especially since the character of the morbid change is absolutely similar to that which Dr. Handfield Jones and Dr. Sutton call fibroid degeneration, and which, when existing in its most extreme form, gives rise to the condition known as Cirrhosis of the Lung. All these fibroid ch anges, therefore, as well as the analogous processes which occur in the lungs of artisans whose air-passages are exposed to the contact and irritation of foreign particles, and the induration which often binds closely packed nodules of tubercle together even where no caverns exist, are intimately related to one another, and are due, I believe, much more to local than to constitutional causes. Because these fibroid indurations or substitutions coexist so frequently with tubercular and socalled scrofulous diseases of the lung, we must not look upon them as necessarily and immediately dependent upon the same constitutional cause. It is well known that precisely the same kind of induration is produced around such an accidental cause of irritation as the existence of an acephalo-cyst in the midst of the lung-tissue, as we find produced around the walls of a tubercular or scrofulous cavern. Mechanical irritation, produced by the inhalation of fine foreign particles, produces the same kind of induration in the lungs of artisans who may be quite free from constitutional tubercular taint; and, as I have stated on a former

* There also seems reason to believe that, much more rarely, the grey granulation may shrink in size, and become organised into more fully formed fibre-tissue. occasion, a very large number of the cases of Cirrhosis of the Lung in its pure form, no tubercle whatever is met with. Such facts should, I think, tend strongly to convince us, not only that fibroid changes may exist alone in the lung, quite independently of any tubercular or scrofulous taint, but also that, when coexisting in the same organ with the products peculiar to either of these constitutional conditions, these changes may even then be secondary and local, rather than primary. They are, in fact, as we so often see, rather conservative in their nature, and to this extent, so far as tendency is concerned, may be considered as directly antagonistic in their action to the destructive course of the more primary products of disease.

This group of morbid changes has been referred to the category of chronic inflammations by some pathologists, whilst others have preferred to speak of them as fibroid degenerations. In my opinion, the process by which this morbid alteration is brought about differs in important respects from inflammation on the one hand, and from degeneration on the other. It seems to occupy an intermediate position, since it simply leads to the formation of a new growth foreign to the parts. Looking upon a characteristic inflammatory process as one in which there is not only a greatly increased formative stimulus leading to a rapid hyperplasia of tissue-elements, but also a rapid necrobiosis or molecular death of the new-formed and old adjacent elements-so that rapid production and rapid death are advancing side by side-the process in question seems to present the formative stimulus and increase, and to be almost entirely wanting in the destructive part of the inflammatory change, since the new-formed elements persist. On the other hand, there is not merely the damage to the actual structural elements of the part, such as we meet with in degenerations (of which the fatty may be taken as the type), but rather the complete disappearance of the functional elements of the organ, whose place is gradually occupied by an advancing new growth. The new growth, it is true, is functionally inert, so that there is an amount of functional degradation of the organ answering to the textural replacement, although there is no degenera. tion of tissue in the strict sense of the term. For these reasons, I would propose that all such tissue-changes should neither be styled chronic inflammations nor degenerations, but, instead, that the process should be spoken of as one of fibroid substitution. This name seems a good one, as it is merely descriptive of what actually takes place, and implies no theory as to the nature of the process by which it has been brought about, concerning which differences of opinion may exist.

\section{SPONTANEOUS AMPUTATION OF THE FOREARM.}

\author{
By J. R. SWANTON, M.D., Bantry.
}

ON the afternoon of Saturday, October 5 th, a boy, aged 7 , was brought to my surgery, having, it was stated, strayed into a field for the purpose of picking blackberries, and while there been brutally attacked by a donkey. He certainly presented a pitiable sight, with scarcely a shred of clothes remaining, the countenance indicating the dreadful fright the little fellow must have had; so much so, as to make him almost insensible to pain.

On examination, the bones of the forearm were discovered to have been fractured; the skin appeared to be bruised, but there was no solution of continuity. From the bloodless appearance of the hand, the father was informed that amputation would be advisable. To this, he decidedly objected. The arm was put up in light splints, well padded with lint. Next morning, on calling at the house, I found the splints removed, and others rather tightly put on. A bone-setter had been called in. The child suffered much pain; had a sleepless night, with raving. Of course I discontinued my attendance, and saw nothing of the case for some days; until, as the child's suffering was increased, and danger apprehended, a dispensary ticket was sought. The medical officer kindly asked me to accompany him. The splints and bandages were forthwith removed; several of the fingers were gangrenous; but pulsation and warmth led to the hope of saving others. The discharge was most offensive.

After some days, the bones of the wrist became exposed, and soon the whole hand had mortified. As the healing process was slow, and the child's constitution not robust, the question of secondary amputation was.
considered, with a view to lessen the suppuration and give a good stump. It was decided, however, to remove with a pliers merely such portion of the bone as projected from the wound, which was accordingly done. The healing progressed favourably, and the child has now a good stump formed of half the forearm. The dressings throughout were of the simplest character, due attention being paid to the support
of the constitution. 